Z. klin. Chem. u. klin. Biochem.

8. Jg., S. $525-528$, September 1970

\title{
Umesterung und Veresterung von lang- und kurzkettigen Fettsäuren in Kapillaren für die Gaschromatographie
}

\author{
Von K. OetTe ${ }^{1}$ ), M. Doss' ${ }^{2}$ ) und M. WINTERFeLD ${ }^{1}$ ) \\ Aus der Medizinischen Universitätsklinik Köln (Direktor: Prof. Dr. R. Gross) und dem Hygiene-Institut der Universität \\ Marburg (Direktor: Prof. Dr. R. Siegert)
}

(Eingegangen am 25. Mai 1970)

\begin{abstract}
E̊ werden Mikromethoden für die Umesterung von Glyceriden und Glycerinphosphatiden sowie die Veresterung von freien Fettsäuren zu Fettsäuremethylestern in Kapillaren beschrieben. Die Reaktionen wurden in einer an ein Schliffröhrchen angesetzten Kapillare und in der Hamilton-Mikroliter-Spritze durchgeführt. Glyceride und Glycerinphosphatide wurden mit Na-Methylat in Methanol-Benzol umgeestert. Die Veresterung freier Fettsäuren erfolgte in ätherischer Diazomethanlösung. In beiden Fällen vollzog sich unter den angegebenen Bedingungen die Reaktion innerhalb weniger Minuten quantitativ.

Die Methoden sind speziell zur quantitativen Analyse flüchtiger Fettsäuremethylester entwickelt worden. Auf weitere Anwendungsbereiche im Vergleich zu bereits veröffentlichten Methoden wird eingegangen.

\section{Transesterification and esterification of long- and short cbain fatty acids in capillaries for gascbromatographic analysis}

Micromethods for the transesterification of glycerides and glycerophosphatides and for the esterification of free fatty acids in capillartubes are described. The reagents used were Na-methylate and diazomethane. The simple and rapid method needs no extraction and was especially developed for the gaschromatographic analysis of volatile fatty acids. Further applications in comparison to the methods already published are described.
\end{abstract}

In vorangehenden Arbeiten (1-3) wurden alkalikatalysierte Umesterungsmethoden zur Darstellung von Fettsäuremethylestern aus Lipiden beschrieben. Die Methoden zeichnen sich durch einfache Technik, schnelle Umsetzungsreaktionen, ihre Anwendbarkeit im Mikrobereich sowie durch verschiedene Kombinationsmöglichkeiten mit der Dünnschichtchromatographie aus. Sie wurden speziell zur gaschromatographischen Fettsäureanalyse bestimmter Lipidklassen $(1-3)$ ausgearbeitet und haben sich u. a. für gaschromatographische Serienanalysen und Fettsäureuntersuchungen (4) in Biopsiematerial bewährt.

Es zeigte sich, daß das früher angegebene Schliffröhrchen mit angesetzter Kapillare (2), dessen Volumen zwischen 20 und $100 \mu l$ variiert, eine weitere wesentliche Verbesserung der Mikromethode zuließ, da bereits kleinste Na-Methylatvolumina zur alkali-katalysierten Umesterung ausreichen. Zusätzliche Experimente ergaben, daß sich hiermit auch freie Fettsäuren mit ätherischer Diazomethanlösung im Mikrolitermaßstab verestern lassen. Die geringe Verdampfungsmöglichkeit in den Kapillaren während der Umund Veresterung bietet ferner die Möglichkeit, die freien oder gebundenen flüchtigen kurzkettigen Fettsäuren ohne wesentliche Verdampfungsverluste in Fettsäuremethylester zu überführen und in die Säule des Gaschromatographen einzuspritzen.

1) $5 \mathrm{Köln-Lindenthal,} \mathrm{Joseph-Stelzmann-Straße} 9$.

2) 355 Marburg an der Lahn, Pilgrimstein 2.

\section{Material und Methoden}

Testsubstanzen

Triglyceride (Maisöl, Leinsamenöl), Glycerinphosphatide (Leber), Cholesterinester (Serum), mittelkettige Triglyceride (Margarine Union $\mathrm{GmbH}$, Hamburg).

Referenzsubstanzen für Fettsäuremethylester (Applied Science Laboratories, Pa., USA, zu beziehen über Serva, Heidelberg).

Freie Fettsäuren wurden aus Maisöl und mittelkettigen Triglyceriden durch Verseifung und Extraktion mit Petroläther $\mathrm{Kp} 40^{\circ}$ bis $60^{\circ}$ nach Ansäuerung gewonnen.

Reagenzien

$0,25 \mathrm{~N} \mathrm{Na}-$ Methylat (1)

$2 \mathrm{~N}$ methanolische $\mathrm{HCl}$ (wasserfrei)

Benzol

Diazomethan in ätherischer Lösung wurde aus Nitrosomethylharnstoff (Fluka AG, Schweiz) durch Einwirkung von 40proz. wäßr. $\mathrm{KOH}$ hergestellt (5). Eine zusätzliche Reinigung erfolgte durch Destillation in eine Vorlage mit Äther.

Dünnschichtchromatographie

Zur Dünnschichtchromatographie wurden Kieselgel-H-Platten (Kieselgel H nach Stahl, E. Merck, Darmstadt) und SelectaKieselgel-Fertigplatten Nr. 1500, säurefest (Schleicher und Schüll, Dassel) verwendet. Das Lösungsmittelsystem zur Trennung von freien Fettsäuren, Triglyceriden, Methylestern, Cholesterin und Cholesterinestern bestand aus Petroläther $\mathrm{Kp} 40^{\circ}-60^{\circ}$ Diäthyläther 9:1 (v/v). Weitere Einzelheiten zur Dünnschichtchromatographie sind in vorangehenden Arbeiten zu finden (1-4).

\section{Gaschromatographie}

Die gaschromatographischen Analysen wurden unter den folgenden Bedingungen mit einem Gerät der Firma Packard Instrument GmbH, Frankfurt am Main, durchgeführt: Säulenfüllung 2,5\% Athylenglykolsuccinat (EGS) auf Chromosorb G (AWDMCS, 80-100 mesh, Perkin-Elmer, Überlingen/Bodensce); 
Trägcrgas Argon, Durchfluß 60-80 m//Min., Inlet Temperatur $200^{\circ}$, Temperatur der Säule $155^{\circ}$, für langkettige Fettsäuremethylester bzw. $70^{\circ}$ für kurz- und mittelkettige Fetțäuremethylester, Temperatur des Outlet und Flammenionisationsdetektors $200^{\circ}$, Detektor-Spannung $500 \mathrm{~V}$, Höchste Empfindlichkeit 3·10-10 Amp.

\section{Arbeitsvorschriften}

Umesterung von Glyceriden und Glycerinphosphatiden Die zur Umesterung bestimmten Substanzen werden in einer Menge von $20-400 \mu \mathrm{g}$ - gelöst in wenigen $\mu l$ Diäthyläther, Aceton, Chloroform oder Benzol - mit einer Mikropipette (z. B. Hamilton-Spritze) in die Kapillarspitze des Reaktionsgefäßes (Abb. 1) (2) überführt. Nach vorsichtigem Abblasen des

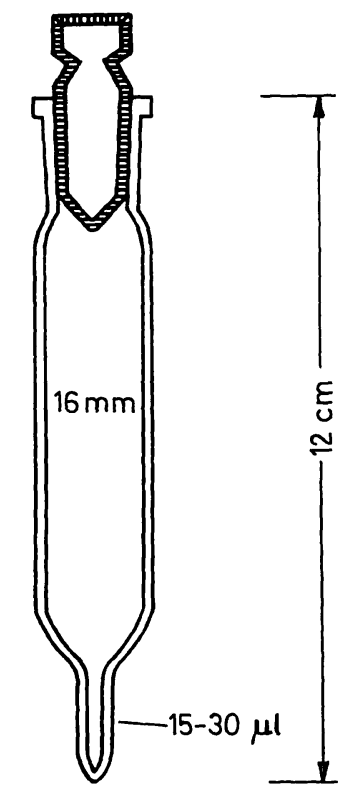

Abb.

Reaktionsgefäß mit Kapillarspitze

Lösungsmittels im Stickstoffstrom mit einer Pasteur-Pipette werden die Lipide mit $2 \mu /$ Benzol von der Kapillarwand gelöst. Die Lösung wird anschließend mit $5 \mu / 0,25 \mathrm{~N} \mathrm{Na-Methylat} \mathrm{ver-}$ setzt. Durch mehrfaches Aufziehen in eine $10 \mu \mathrm{l}$ HamiltonSpritze erfolgt das notwendige Mischen und Lösen. Kurzzeitiges (etwa 1 Min.) Eintauchen der Kapillarspitze des Reaktionsgefäßes in ein auf $60-70^{\circ}$ temperiertes Wasserbad beschleunigt den Vorgang. Nach $5 \mathrm{Min}$. wird das Gemisch in der Kapillarspitze des Reaktionsgefäßes mit $1 \mu / 2 \mathrm{~N}$ Methanol-HCl unter Verwendung einer Hamilton-Spritze neutralisiert (Säureüberschuß). Das Gemisch ist fertig zur gaschromatographischen Analyse. Je nach der Empfindlichkeit des Detektors werden zwischen 5 und $25 \mu \mathrm{g}$ Fettsäuremethylester injiziert, deren Lösungsvolumen möglichst nur 0,5 bis $2,0 \mu l$ betragen soll.

Die Umesterung von Lipiden mit kürzerkettigen, leichterflüchtigen Fettsäuren erfolgt unter milderen Bedingungen in der Kapillare einer $10 \mu /$ Hamilton-Spritze nach Mischung der in Benzol gelösten Lipide mit Na-Methylat. Hierfür wird nur zweibis dreimal aufgezogen (s. o.). Die Umesterung vollzieht sich ebenfalls innerhalb von 5 Min. Zur Neutralisation des Reaktionsgemisches wird zunächst $1 \mu \mathrm{l} 2 \mathrm{~N}$ methanolische $\mathrm{HCl}$ in die $\mathrm{Ka}$ pillarspitze des Reaktionsgefäßes deponiert. Anschließend stellt man das Gefäß in Eiswasser, injiziert das Gemisch in die gekühlte methanolische $\mathrm{HCl}$ und neutralisiert durch schnelles mehrfaches Einsaugen und Ausspritzen innerhalb weniger Sek. Danach wird das Gemisch sofort in die Hamilton-Spritze eingesaugt und kann nunmehr zur gaschromatographischen Analyse verwendet werden.

Veresterung von freien Fettsäuren

Die Füllung der Kapillarspitze des Reaktionsgefäßes mit freien Fettsäuren $(20-400 \mu \mathrm{g})$ erfolgt wie oben beschrieben. Nach
Abblasen des Lösungsmittels im Stickstoffstrom werden 10-20 $\mu$ l einer intensiv gelb gefärbten, eisgekühlten ätherischen Diazomethanlösung unter Beobachtung hinzugegeben. Die Veresterung wird im Eisbad vorgenommen. Dabei darf sich die Diazomethanlösung im Verlauf von etwa 2 Min. nur teilweise entfärben. Exst dann ist die vollständige Veresterung gewährleistet. Die Probe ist damit zur gaschromatographischen Analyse vorbereitet. Das Injektionsvolumen sollte $2 \mu l$ nicht überschreiten. Eine eventuell notwendige Konzentrierung der Methylester erfolgt durch Einengung der Lösung im Stickstoffstrom. - Die Kapillarspitze läßt sich durch Ausglühen reinigen.

\section{Ergebnisse und Diskussion}

Wie bereits früher mitgeteilt wurde (1-3), können mit der alkalikatalysierten Umesterung durch $\mathrm{Na}$-Methylat Glyceride und Glycerinphosphatide neben einigen anderen Stoffklassen schnell und quantitativ zu Fettsäuremethylestern umgesetzt werden. Das mit Hilfe der Kapillartechnik verbesserte Verfahren erspart unter Beibehaltung der Vorteile gegenüber der säurekatalysierten Umesterung sowohl die Extraktion (1) als auch die Elution $(2,3)$. Durch die Einbeziehung der Veresterung von freien Fettsäuren mit Diazomethan (Abb. 2) und der Um- und Veresterung von kürzer-

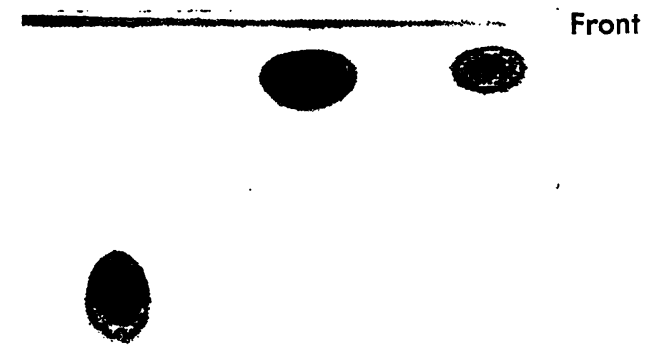

Front

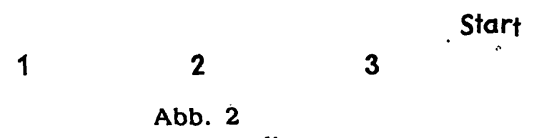

Dünnschichtchromatographische Darstellung freier und mit Diazomethan in der Kapillare veresterter Olsäure auf Selecta-Kieselgelplatten

Laufmittel: Äther: Petroläther $\left(\mathrm{Kp} 40^{\circ}-60^{\circ}\right)$ : Eisessig $(28: 70: 2, \mathrm{v} / \mathrm{v})$ Platte: Selecta-Kieselgel-Fertigplatte; Darstellung siehe Abbildung 1 . 1. Ölsäure

. Olsäuremethylester nach Veresterung mit der Kapillarmethode 3. Fettsäuremethylester (Testmischung).

kettigen Fettsäuren wurde der Anwendungsbereich der Methodik erweitert. Die Tabellen $1 \mathrm{a}$ und $\mathrm{b}$ zeigen einen Vergleich zwischen den einzelnen Methoden am Beispiel der gaschromatographischen Fettsäureanalyse von Mais- und Leinsamenöl sowie mittelkettigem Triglycerid-Öl. Die Ergebnisse der Umesterungmethoden liegen bei den Glyceriden langkettiger Fettsäuren (Tab. 1 b) innerhalb der für die gaschromatographische Analyse bekannten Fehlergrenzen (6). Die Analyse des mittelkettigen Triglycerid-Öls (Tab. 1a) weist auf einen geringen Verdampfungsverlust bei Verwendung der dünnsschichtchromatographischen $\mathrm{Me}$ - 
Tab. 1

Vergleich alkali-katalysierter Umesterungsmethoden im Mikrobereich (A Dünnschichtchromatographische- (2) und B Kapillarmethode) mit der Veresterung aus den gleichen Glyceriden freigesetzter Fettsäuren durch Diazomethan (C Kapillarmethode). MKT = mittelkettige Tryglyceride

a) Gaschromatographische Analyse mittelkettiger Fettsäuregemische*)

\begin{tabular}{|c|c|c|c|c|c|c|}
\hline $\begin{array}{l}\text { Fettsäure- } \\
\text { methylester }\end{array}$ & $\mathrm{A}^{M}$ & $-\operatorname{Mar}_{\mathbf{B}}$ & c & A & $M_{\mathrm{B}} \mathrm{KTI}^{-\circlearrowleft I}$ & C \\
\hline $\begin{array}{r}C_{8: 0} \\
C_{10: 0}\end{array}$ & $\begin{array}{l}18,0 \\
82,0\end{array}$ & $\begin{array}{l}21,5 \\
78,5\end{array}$ & $\begin{array}{l}20,6 \\
79,4\end{array}$ & $\begin{array}{l}45,5 \\
54,5\end{array}$ & $\begin{array}{l}48,5 \\
51,5\end{array}$ & $\begin{array}{l}50,0 \\
50,0\end{array}$ \\
\hline
\end{tabular}

b) Gaschromatographische Analyse längerkettiger Fettsäuregemische*)

\begin{tabular}{crrrrrr}
\hline $\begin{array}{c}\text { Fettsäure- } \\
\text { methylester }\end{array}$ & A & $\begin{array}{c}\text { Mazola-Ol } \\
\text { B }\end{array}$ & C & A & Leinöl & B \\
\hline$C_{16: 0}$ & 13,0 & 11,6 & 13,6 & 6,3 & 7,1 & 6,5 \\
$C_{18: 0}$ & 3,0 & 2,8 & 4,2 & 4,9 & 6,1 & 6,1 \\
$C_{18: 1}$ & 32,0 & 30,5 & 32,8 & 17,4 & 17,4 & 19,7 \\
$C_{18: 2}$ & 50,5 & 53,4 & 47,8 & 19,2 & 20,8 & 21,1 \\
$C_{18}$ & 1,5 & 1,7 & 1,6 & 52,2 & 48,6 & 46,6 \\
\hline
\end{tabular}

*) In geringer Menge (unter $1 \%$ ) nachweisbare kürzer- bzw. längerkettige Komponenten wurden in die Berechnung nicht einbezogen

thode hin. Die Unterschiede zwischen den Analysen der veresterten und umgeesterten Fettsäuren schließen die durch Verseifung entstandenen Fehler mit ein.

Cholesterinester, die schon in früheren Experimenten (1) als die für die alkali-katalysierte Umesterung kritische Lipidklasse ermittelt wurden, konnten trotz guter Lösung bei Zimmertemperatur in der Kapillare nicht vollständig umgesetzt werden. Bei Verlängerung der Reaktionszeit auf 30 Min. und Erhöhung der Methylat-Konzentration auf $0,5 \mathrm{~N}$ wurden bei einem Benzol/Na-Methylat-Verhältnis von $1: 2(\mathrm{v} / \mathrm{v})$ über $50 \%$ Cholesterinoleat umgeestert. Kleinere Anteile von Cholesterinestern im Lipidgemisch werden allerdings auch mit der Kapillartechnik quantitativ umgesetzt, wie es für die Umesterungsreaktionen mit größeren Volumina beschrieben wurde $(1,2)$.

In Anlehnung an die bereits veröffentlichten Methoden wurde auch für das Kapillarverfahren eine Umesterungszeit von 5 Min. gewählt. Das Dünnschichtchromatogramm der Abbildung 3 zeigt die vollständige Umesterung von Triglyceriden unter den in der Arbeitsvorschrift angegebenen Bedingungen.

Aus Zeitstudien ging hervor, $\mathrm{da} \beta$ sowohl nach 10 als auch nach 15 Min. keine nennenswerte Seifenbildung unter der Einwirkung von Na-Methylat eingetreten war. Die Bildung von Seifen aus esterartig gebundenen Fettsäuren kommt bei der alkali-katalysierten Umesterung erst mit höheren $\mathrm{Na}$-Methylat-Konzentrationen und bei höheren Temperaturen in Gang. Vorversuche zeigten, daß ohne Neutralisation des Reaktionsgemisches die gaschromatographische Ausbeute an Fettsäuremethÿlestern deutlich verringert ist. Man muß annehmen, daß bei der Injektion des nicht neutralisierten Reaktionsgemisches eine Verseifung und Salzbildung im . Bereich der höher temperierten Verdampfungszone des Gaschromatographen eintritt. Erst nach Neutralisation des Reaktionsgemisches mit methanolischer Salzsäure war die gaschromatographische Empfindlichkeit vorausberechenbar und die Analyse

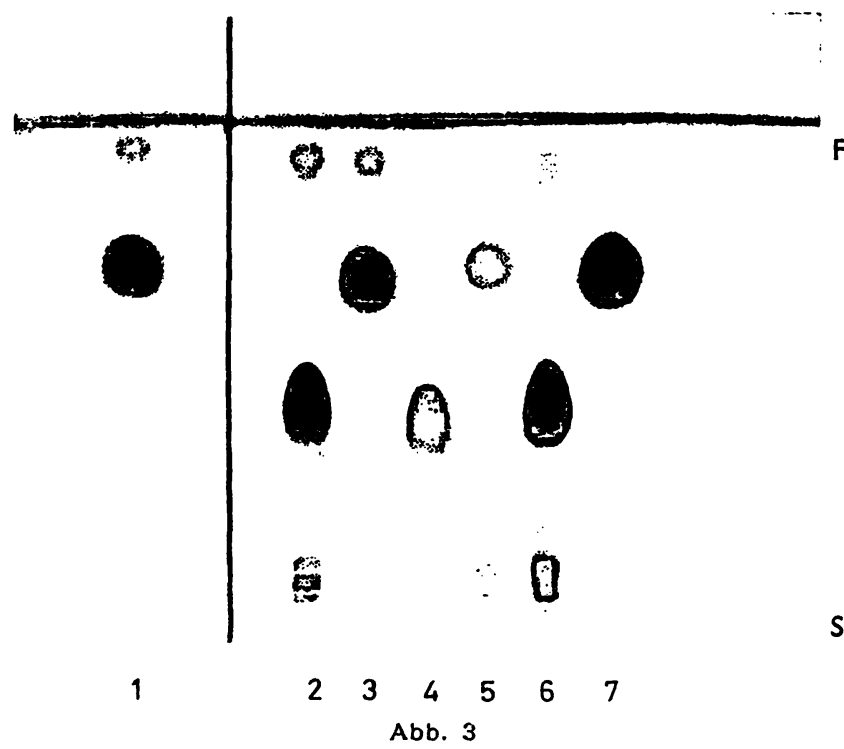

Kieselgel-H-Dünnschichtchromatogramm mit Fettsäuremethylestern und Triglyceriden, entwickelt im Lösungsmittelsystem Petroläther (Kp $\left.40^{\circ}-60^{\circ}\right)$-Diäthyläther $(9: 1, v / v)$. Darstellung durch Besprühen mit 50proz. wäßr. Schwefelsäure und ${ }_{180^{\circ}}$ Erhitzen der Kieselgelplatte auf
mita

1. Fettsäuremethylester aus Maisöl nach Umesterung auf der Kieselgel-H-Platte mit in Na-Methylat

2. Maisöl

3. Fettsäuremethylester aus Maisöl nach Umesterung mit $0,25 \mathrm{~N}$ Na-Methylat in der Kapillare

4. MKT-Ö

5. Fettsäuremethylester aus $M K T-O I$ nach Umesterung mit $0,25 \mathrm{~N}$ Na-Methylat in der Kapillare

6. Referenz-Triglyceride

7. Oisäuremethylester.

Am und direkt über dem Start (S) bei 2,5 und 6 Spuren freier Fettsäuren und von Mono- und Diglyceriden $M K T=$ Mittelkettige Triglyceride $\mathrm{F}=$ Front

reproduzierbar. Das Fettsäuremethylestergemisch wurde in schwachsaurem Methanol-Benzol auf die Säule appliziert.

Bei kleinen Lipidmengen war die Umesterung in der Kapillare oder in der Spritze von besonderem Vorteil, $\mathrm{da}$ das Reaktionsgemisch keine die gaschromatographische Analyse störenden Verunreinigungen enthielt. Allerdings muß darauf geachtet werden, da $\beta$ das Verhältnis zwischen Lipidmenge und Injektionsvolumen so gewählt wird, daß der Lösungsmittelpeak klein bleibt und keine Substanzen mit kurzer Retentionszeit verschluckt.

Ein Vorteil der alkali-katalysierten Umesterung in Kapillaren besteht in ihrer Anwendung auf flüchtige Methylester. Durch die Umesterung in der Kapillare wird ein Verlust von Methylestern durçh Verdampfen bei Raumtemperatur weitgehend vermieden. Die Technik der Neutralisation gestattet ebenfalls eine praktisch verlustlose Aufarbeitung bis zur Injektion des Gemisches in die Säule des Gaschromatographen. $\mathrm{Zu}$ beachten ist, daß für die Analyse von Fettsäuremethylestern mit weniger als sechs C-Atomen ein Flammenionisationsdetektor unerläßlich ist, da der ArgonIonisationsdetektor niedermolekulare Substanzen nicht mehr linear registriert.

Bei einer vergleichenden Beurteilung der Kapillartechnik mit den Umesterungsmethoden im Reaktionsgefäß $(1,2)$ und auf der Kieselgel-H-Dünnschichtplatte $(2,3)$ sind folgende Punkte hervorzuheben: 
Cholesterinester können nur im Reaktionsgefäß quantitativ umgeestert werden. Die Vorzüge der alkalikatalysierten Umesterung von Triglyceriden und Glycerinphosphatiden auf Kieselgelschichten bestehen in der Kombination mit der gaschromatographischen Analyse von Lipiden. Die Methode läßt sich für Serienbestimmungen verwenden. Die Kapillartechnik eignet sich besonders für die Umesterung flüchtiger Fettsäuren, läßt sich aber auch für die Veresterung mit Diazomethan im Mikromaßstab bei Serienbestimmungen vorteilhaft einsetzen. Während der gaschromatographische Leerwert bei der Umesterung auf Kieselgel-HPlatten besondere Aufmerksamkeit verlangt, wurden im Gaschromatogramm der Fettsäuremethylester nach Injektion des gesamten Reaktionsgemisches aus der Kapillare keine interferierenden Substanzen beobachtet.
Abschließend soll eine Veröffentlichung von HušeK (7) Erwähnung finden, der an Kieselgel gebundene Lipide ohne Extraktion im Reaktionsgefäß umesterte. In Verbindung mit der Dünnschichtchromatographie wurden bereits in einer vorangehenden Arbeit die Umesterung von an'Kieselgel gebundenen Lipiden und deren Vorteile beschrieben $(2 ; 3)$. Betont muß werden, $\mathrm{da} ß$. die Umesterung im Reaktionsgefäß nur noch für die Cholesterinester sinnvoll ist. Hierfür kann bei Anwendung der Dünnschichtchromatographie eines der von uns mitgeteilten Standardverfahren (1,2 und 3) verwendet werden, evtl. unter direkter Zugabe des mit Cholesterinester beladenen Kieselgels (7) zum Lösungsmittel, z. B. Benzol. Erst dann läßt man das NaMethylat mit dem Lipid reagieren.

Die Untersuchungen wurden in dankenswerter Weise durch Mittel der Deutschen Forschungsgemeinschaft unterstützt.

\section{Literatur}

1. Doss, M. und K. Oetre, diese Z. 3, 125 (1965). - 2. Oette, K. und M. Doss, J. Chromatog. 32, 439 (1968). - 3. Doss, M. und K. Oetre, Z. analyt. Chem. 243, 350 (1968). - 4. Oetre, K. und R. Phlippen, Verh. dtsch. Ges. inn. Med. 74, 247 (1968). 5. ZölLNER, N. und D. EBERHAGEN, In: Untersuchung und Be- stimmung der Lipide im Blut, Springer-Verlag, Berlin-HeidelbergNew York (1965). - 6. Horning, E. C., E. H. Ahrens, Jr., S. R. Lipsky, F. H. Mattson, J. F. Mead, D. A. Turner und W. H. Goldwater, J. Lipid Res. 5, 20 (1964). - 7. HušEk, P., diese Z. 7, 627 (1969).
Priv.-Doz. Dr. Kurt Oette $5 \mathrm{Köln}$-Lindenthal Joseph-Stelzmann-Str. 9 